\title{
The Phase-Il ATLAS ITk Pixel Upgrade
}

\author{
S. Terzo ${ }^{a *}$ on behalf of the ATLAS Collaboration \\ a Institut de Física d'Altes Energies (IFAE), The Barcelona Institute of Science and Technology \\ Edifici Cn, UAB campus, E-08193 Bellaterra (Barcelona), Spain \\ E-mail: Stefano.Terzodcern.ch
}

ABSTRACT: The entire tracking system of the ATLAS experiment will be replaced during the LHC Phase-II shutdown (foreseen to take place around 2025) by an all-silicon detector called the "ITk" (Inner Tracker). The innermost portion of ITk will consist of a pixel detector with five layers in the barrel region and ring-shaped supports in the end-cap regions. It will be instrumented with new sensor and readout electronics technologies to improve the tracking performance and cope with the HL-LHC environment, which will be severe in terms of occupancy and radiation levels. The new pixel system could include up to $14 \mathrm{~m}^{2}$ of silicon, depending on the final layout, which is expected to be decided in 2017. Several layout options are being investigated at the moment, including some with novel inclined support structures in the barrel end-cap overlap region and others with very long innermost barrel layers. Forward coverage could be as high as letal $<4$. Supporting structures will be based on low mass, highly stable and highly thermally conductive carbon-based materials cooled by evaporative carbon dioxide circulated in thin-walled titanium pipes embedded in the structures. Planar, 3D, and CMOS sensors are being investigated to identify the optimal technology, which may be different for the various layers. The RD53 Collaboration is developing the new readout chip. The pixel off-detector readout electronics will be implemented in the framework of the general ATLAS trigger and DAQ system. A readout speed of up to $5 \mathrm{~Gb} / \mathrm{s}$ per data link will be needed in the innermost layers going down to $640 \mathrm{Mb} / \mathrm{s}$ for the outermost. Because of the very high radiation level inside the detector, the first part of the transmission has to be implemented electrically, with signals converted for optical transmission at larger radii. Extensive tests are being carried out to prove the feasibility of implementing serial powering, which has been chosen as the baseline for the ITk pixel system due to the reduced material in the servicing cables foreseen for this option.

KEYWORDS: Tracker; Pixel detector; HL-LHC; ATLAS; ITk.

\footnotetext{
${ }^{*}$ Corresponding author.
} 


\section{Contents}

1. Introduction 目

2. ITk layout and mechanics

2.1] The pixel detector layout

3. Pixel modules 3

3.1 Front-end chip 3

3.2 Possible sensor technologies

母. Electrical services

5. Conclusions 6

\section{Introduction}

An upgrade of the Large Hadron Collider (LHC) is planned around 2025 to increase the accelerator luminosity by a factor of 5-7 with respect to the nominal value of $10^{34} \mathrm{~cm}^{-2} \mathrm{~s}^{-1}$. In the following High Luminosity phase of the LHC (HL-LHC) [四] about 140-200 proton collisions per bunch crossing are expected. During the same long shutdown period the ATLAS detector [2] will undergo major upgrades required to face the high particle multiplicity, large pile-up and the huge radiation doses at HL-LHC. In particular the whole Inner Detector (ID) will be replaced by a new Inner Tracker (ITk) fully composed of silicon devices which will require a finer granularity, especially close to the interaction point. The main challenge for pixel modules to maintain tracking and b-tagging performance during the full HL-LHC data taking will be the radiation damage due to particle fluences which will reach up to about $2 \times 10^{16} \mathrm{n}_{\mathrm{eq}} / \mathrm{cm}^{2}$ for the innermost pixel layer.

\section{ITk layout and mechanics}

The ITk layout will consist of a strip detector made of four barrel layers and six end-caps per side at a distance larger than $362 \mathrm{~mm}$ from the beam line, and a pixel detector with five barrel layers from a radius of about $39 \mathrm{~mm}$ to about $271 \mathrm{~mm}$. The latter will be completed by a forward structure of concentric rings arranged at different radii. Due to the harsh radiation levels close to the beam line which may be beyond the pixel module capabilities, the option of making the two innermost pixel barrel layers replaceable to maintain the detector performance over the full HL-LHC run period is being considered. The full surface foreseen for pixel detector will be of about $14 \mathrm{~m}^{2}$. 


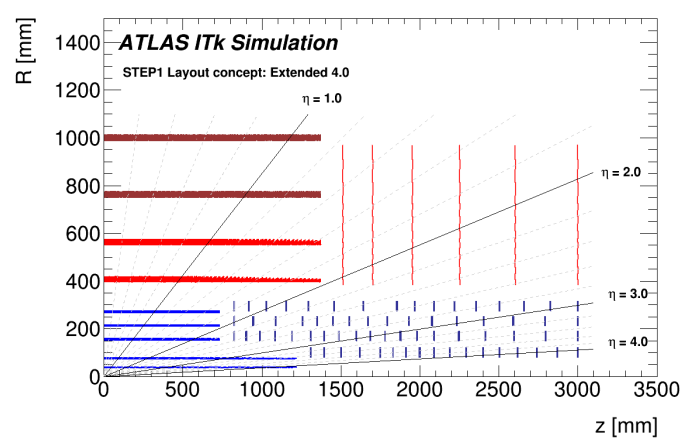

(a) Extended $-\eta<|4|$

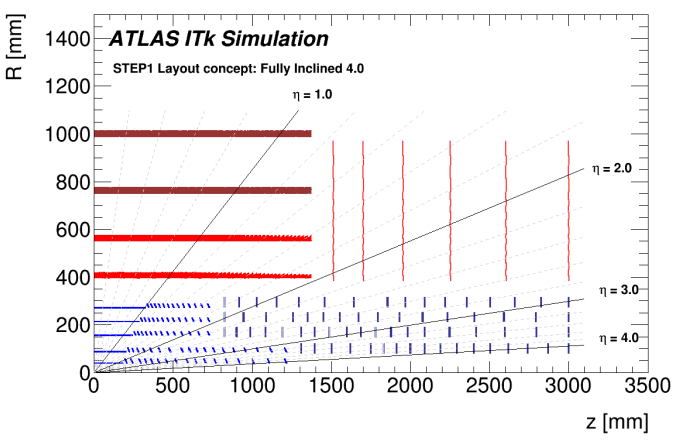

(b) Fully inclined $-\eta<|4|$

Figure 1. Two possible layouts for the ITk [3]. In each picture a quarter of the detector is represented where the strips are shown in red and the pixel detectors in blue. In a the extended layout with a pseudo-rapidity coverage of the barrel up to $\eta=|4|$ is shown. In (b) the fully inclined layout is shown. The pseudo-rapidity coverage of this layout is also up to $\eta=|4|$.

\subsection{The pixel detector layout}

Two different layout concepts of the ITk pixel detector are presently under investigation which are shown in Figure [1: an extended layout and an inclined layout [ [4]. To improve the pile-up rejection in the dense track environment at HL-LHC both layouts foresee an increase of the pseudo-rapidity coverage of the barrel with respect to the present ID.

Extended layout. In the extended layout the larger coverage is obtained increasing the length along the beam line of one or both the two innermost barrel layers as shown in Figure 1(a) achieving a pseudo-rapidity coverage of up to $\eta=|3.2|$ or $\eta=|4|$, respectively. With this layout at large pseudo-rapidity, particles will cross several pixels along their length creating long clusters. On one hand this will result in an increased occupancy mostly dependent on the active thickness of the pixel sensors, but on the other hand such a configuration could be used for standalone tracking within one single layer. With long clusters it is indeed possible to extract the information about the entrance and the exit point of the particles with a precision that depends on the pixel size along the beam axis, and improve the matching of the track to the corresponding proton collision. To fully exploit this feature the probability of split clusters due to detector inefficiencies will have to be considered especially after high radiation doses. This layout allows to keep a design of the stave mechanics similar to the present one, but for a larger impact on the material budget in the forward regions. For the extended layout the "I-beam" design shown in Figure 2 has been proposed. This consist of low mass support structures coupling up to two pixel layers with different widths, adaptable distances and tilting angle for the modules. In this solution, electrical services and the pipes of the evaporative $\mathrm{CO}_{2}$ cooling are planned to run within the structure.

Inclined layout. The same large pseudo-rapidity coverage of $\eta \leq|4|$ is obtained tilting the pixel modules at the end of the staves of the two innermost barrel layers or for all the pixel layers as in the fully inclined layout in Figure [1(b). The design of the inclined staves is such that the particles at large pseudo-rapidity will cross the pixel sensor surface with a similar incident angle as for the modules in the central part of the barrel, thus avoiding long clusters. This will result in a reduction 

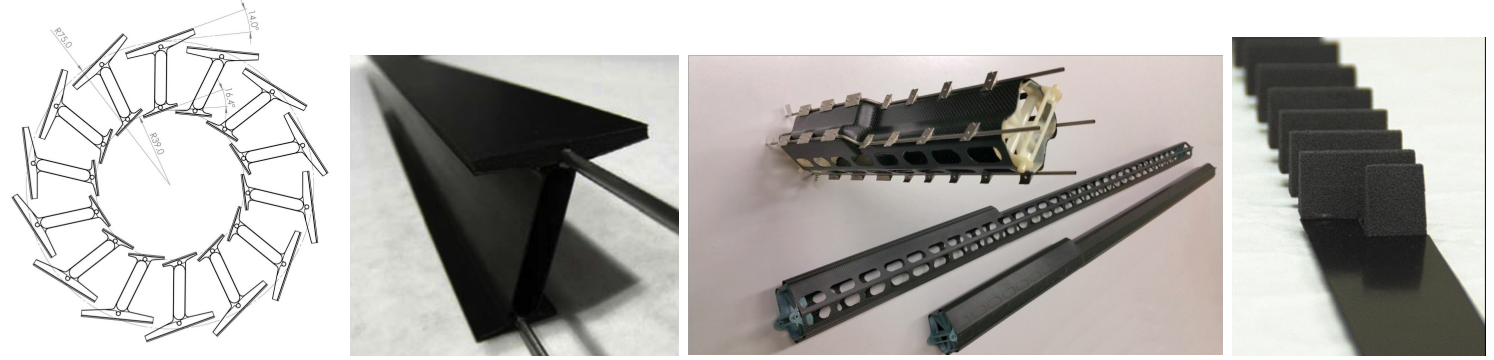

Figure 2. Mechanic prototypes for the different ITk barrel stave layouts. From left to right: the structure of the "I-beam" stave layers for the extended layout; a prototype of the "I-beam" stave; the "slim" stave prototype for the inclined layout; the "alpine" stave prototype, also an alternative for the inclined layout.

of the occupancy and of the material budget at the same time. Moreover the inlined design of the stave will allow to increase the number of points per particle track close to the interaction point in the forward region thus providing a better constraint for the impact parameter resolution. For the inclined layout different designs have been proposed. The prototypes of possible stave supports and mechanics developed can be seen in Figure 2. For inclined pixel sensors the cooling is particularly challenging due to the larger distance of the cooling pipes from the sensors. The titanium cooling pipes carrying the $\mathrm{CO}_{2}$ are placed in the stave base and a carbon foam wedge has to provide both structural support and the sufficient thermal conductivity for the tilted pixel modules attached.

End-cap rings. For the forward region of the ITk, instead of the common disk design used in the present ID, a novel and more flexible ring structure has been designed. This consists of narrow ring shaped supports joined in four concentrical cylindrical layers where each ring can be positioned at a different distance along the beam line. Optimising the pattern of the rings, such a structure allows to provide a constant number of hits for particle tracks in the forward region contributing to the extension of the coverage towards larger pseudo-rapidity. Each ring is built out of two half-rings with a carbon core containing cooling and electrical services. The pixel modules are mounted on both sides of the rings to allow for overlap of the inactive regions. The number of rings per layer will be chosen to ensure at least nine points per track.

\section{Pixel modules}

Different pixel detector technologies are being investigated due to the different radiation levels as a function of the distance from the beam line. The module assembly and production costs for the area to be covered in the different layers are also considered. A small pixel size is necessary in particular close to the interaction point to cope with the increased particle multiplicity.

\subsection{Front-end chip}

A new Front End (FE) chip for the hybrid pixel modules of ITk is bein developed to withstand the harsh radiation fluence and the high particle multiplicity at HL-LHC. The final ITk chip will be produced in $65 \mathrm{~nm}$ CMOS process and derived from a prototype developed by the RD53 Collaboration at CERN [5] as a common effort for the ATLAS and CMS upgrades. The dimensions of the 
new chip will be approximately $(2 \times 2) \mathrm{cm}^{2}$ and its main features will include a smaller pixel size of $(50 \times 50) \mu \mathrm{m}^{2}$ implying a larger number of readout channels with respect to the present chips, and a radiation hardness specified up to $500 \mathrm{MRad}$. In addition it will enable to reach thresholds lower than 1 ke allowing to deal with smaller signals and thus to push further the sensor technology with thinner active substrates. The first version of the RD53 chip, the RD53-A, is under development and the distribution for full module testing is foreseen by the end of 2017. A first small prototype developed to test the analog FE part of this chip, called FE65-P2, has been in the meanwhile characterised demonstrating the possibility to achieve thresholds as low as $500 \mathrm{e}$ to detect particles within $25 \mathrm{~ns}$ with general good performance even after irradiation [6].

\subsection{Possible sensor technologies}

The future ITk chip will be compatible with both $(50 \times 50) \mu \mathrm{m}^{2}$ and $(25 \times 100) \mu \mathrm{m}^{2}$ sensor pixel sizes. The reduced pixel dimension will have the additional advantage of a better position resolution and in the case of 3D sensors also an enhanced radiation hardness.

3D. The 3D sensor technology is already employed in the Insertable B-Layer (IBL) of the present ID and in the ATLAS Forward Proton (AFP) experiment. It consists of an active bulk of p-type silicon into which a reticle of $\mathrm{n}$-doped and p-doped column shaped electrodes are etched perpendicularly to the sensor surface. As opposite to the usual planar sensor design where the electrodes are implanted on the opposite surfaces of the active bulk, in 3D sensors the charge collection distance is decoupled from the active thickness and thus from the number of electron-hole pairs created by a Minimum Ionising Particles (MIP) passing through the active bulk. This allows to reduce the electrode distance without reducing the amount of charge created by MIPs resulting in a low operational voltage and trapping probability after high irradiation fluences. As shown in Figure 3 for the case of the IBL design, 3D sensors need less than $180 \mathrm{~V}$ to reach a hit efficiency similar to the one before irradiation of more than $97 \%$ with perpendicular incident particles, even after irradiation up to $9 \times 10^{15} \mathrm{n}_{\mathrm{eq}} / \mathrm{cm}^{2}$ [ [7]. At these operational voltages the power dissipation is normally less than $15 \mathrm{~mW} / \mathrm{cm}^{2}$. As demonstrated in Ref. [8] for the first prototypes assembled to FE-I4 chips, the performance of 3D detectors is expected to improve with the smaller pixel size foreseen for the ITk due to the consequent further reduction of the electrode distance. The production of 3D devices on the other hand requires a complicated process affecting the yield, the production time and the costs which scales with the number of column electrodes. 3D sensors are thus optimal candidates for the innermost layers where the radiation hardness requirements are more severe but the area to be covered relatively small.

Planar. The n-in-p technology has been chosen as a baseline for planar sensors in the ITk since it is potentially cost-effective due to the only single side processing needed as opposite to the present n-in-n sensor technology employed in the current ATLAS pixel detector. Depending on the required radiation hardness the active thickness can be adjusted to reduce the operational voltage necessary to optimise both hit efficiency and the power dissipation. For the outer barrel layers and the end caps a thickness of $150 \mu \mathrm{m}$ is foreseen while for the innermost layers n-in-p planar pixel sensor with a thickness of $100 \mu \mathrm{m}$ have shown the best performance in terms of charge collection, hit efficiency and power dissipation after irradiation to the HL-LHC fluences as demonstrated in 


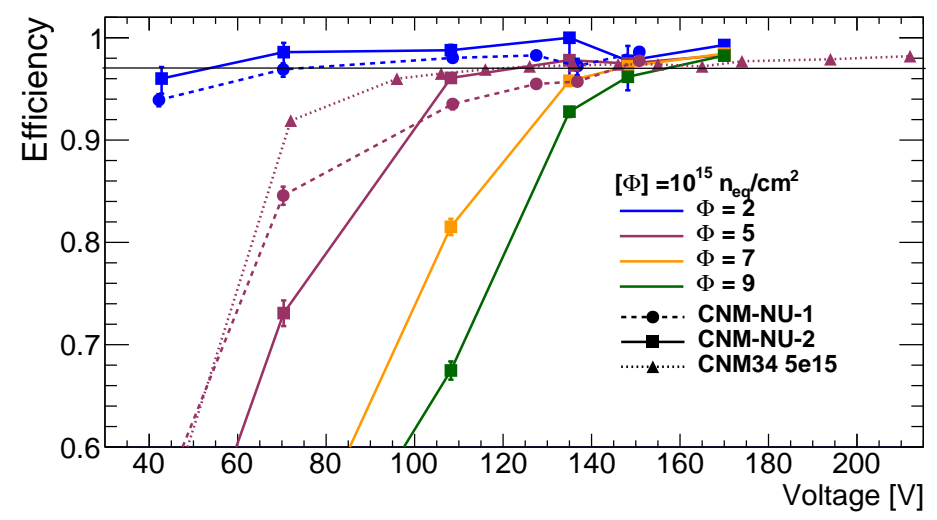

Figure 3. Hit efficiency as a function of the bias voltage of IBL-like 3D pixel sensors irradiated with protons up to a fluence of $9 \times 10^{15} \mathrm{n}_{\mathrm{eq}} \mathrm{cm}^{-2}$. Three modules produced at CNM and assembled to ATLAS FE-I4 chips were employed for these studies and are indicated with different symbols. The different colours represent different irradiation fluences. The efficiency was measured in beam tests performed at CERN SpS with $120 \mathrm{GeV}$ pions impinging perpendicularly to the pixel surface. The solid black line represents the target efficiency of $97 \%$.

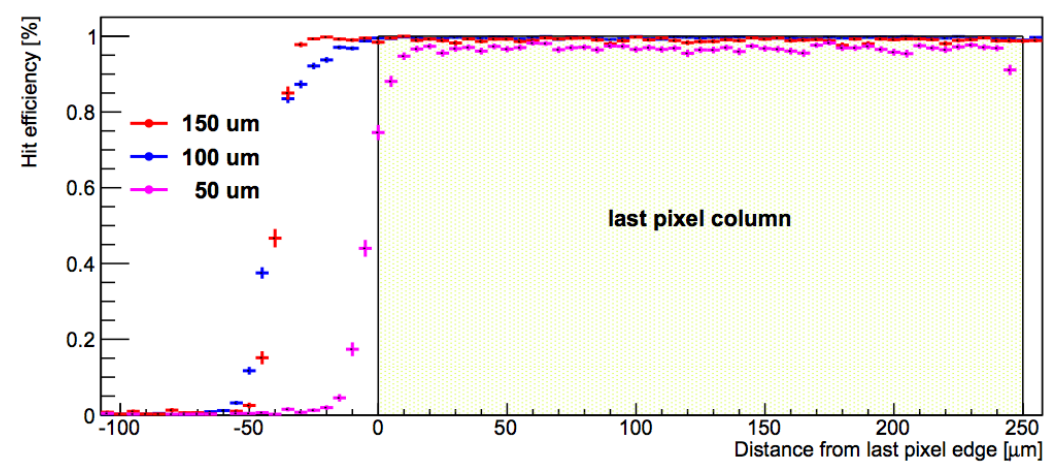

Figure 4. Hit efficiency as a function of the distance from the end of the last pixel implant for n-in-p active edge pixel sensors without bias ring and with floating guard ring structures produced at VTT [10]. The different colours indicate sensors with different active substrate thickness. The nominal implanted cutting edge is at $-50 \mu \mathrm{m}$.

Ref. [9]. Moreover, to employ planar sensors in the innermost layers of ITk where limited overlap space imposes minimal inactive areas at the periphery of the modules, active edge sensor are also being investigated. Such devices are obtained extending the backside implantation to the edge of the sensor allowing to reduce the guard ring structure. This technology allows to produce slim edge planar sensors with a small inactive area as low as $50 \mu \mathrm{m}$ at the periphery of the sensor, and even fully active edge sensors, depending on the active thickness, if no bias ring nor floating guard rings are used, as shown in Figure $\$$.

HR/HV-CMOS. High Resistivity and High Voltage CMOS devices are investigated as an option for the large area to be covered in the fifth barrel layer of the ITk, due to the cost benefits coming from the industrial process [11]. This technology can be employed to produce cost-effective pas- 
sive sensors to be interconnected to ITk readout chips with the usual bump bonding techniques or active pixel sensor embedding a first amplification stage which would allow for potentially cheaper capacitive coupled interconnection with thin glue layers. The most effective solution would be obtained with fully Monolithic Active Pixel Sensors (MAPS) which will completely remove the interconnection costs. First CMOS detector prototypes investigating these three options are presently investigated to prove the radiation hardness and the feasibility of fulfilling all the ATLAS requirements, in particular the $25 \mathrm{~ns}$ time resolution and the power consumption.

\section{Electrical services}

The largest source of multiple scattering in the current ITk layouts comes from cooling pipes and electrical services connecting the end-cap layers to the outer boundaries of the ITk volume. The huge number of modules in the pixel detector would require a big amount of cables with an increased cross section with respect to the current system due to the higher power consumption of the new FE. This implies an increase of the material budget with an impact on the tracking performance. This effort will be reduced in the ITk by using a serial powering scheme consisting of a chain of modules powered with a constant current source. The power can thus be transmitted at low current and high voltage between the modules. Due to the high radiation levels expected inside the detector the data transmission links will be split in an electrical part which will bring the signal from the modules to an end-plate region with opto components, and an optical part taking the data out of the detector. A $5 \mathrm{Gbps}$ data read-out rate is required to fully exploit the performance of the new FE chip. To achieve this, twin-axial or flex cables are investigated. While twin-axials are simpler to produce, flexes contain less material and will thus reduce the multiple scattering. These technologies have been tested and can achieve a data rate of $6.25 \mathrm{Gbps}$.

\section{Conclusions}

The final ITk layout of the pixel detector for the ATLAS Phase-II is under discussion. It will consist of five pixel barrel layers with an extended pseudo-rapidity coverage. A new readout chip is under development and will have an increased granularity and radiation hardness up to $500 \mathrm{MRad}$. The sensor technologies under investigations are $100 \mu \mathrm{m}$ thin planar and 3D silicon sensors for the innermost layers, planar sensors with a thickness of $150 \mu \mathrm{m}$ for the intermediate and outer layers. HV/HR-CMOS pixel sensors are also considered as an option for the fifth layer. According to simulations the full ITk will have a significant reduction in material budget compared to present ID.

\section{References}

[1] O. Brüning, L. Rossi et al., High Luminosity Large Hadron Collider: A description for the European Strategy Preparatory Group, Tech. Rep. CERN-ATS-2012-236 (2012).

[2] ATLAS Collaboration, The ATLAS Experiment at the CERN Large Hadron Collider, JINST 3 (2008) S08003.

[3] B. Smart, ATLAS Pixel Detector Design For HL-LHC, JINST 12 (2017) C02011. 
[4] ATLAS Collaboration, Technical Design Report for the ATLAS Inner Tracker Strip Detector, (2017) ATLAS-TDR-025.

[5] The RD53 Collaboration: https://rd53.web.cern.ch/RD53/

[6] T. Heim et al., FE65-P2: a Prototype Pixel Readout Chip in 65nm Technology for HL-LHC Upgrades, submitted to 2016 IEEE Nucl. Sci. Symp. Conf. Rec.

[7] J. Lange et al., 3D silicon pixel detectors for the High-Luminosity LHC JINST 11 (2016) C01024.

[8] D. Vázquez Fureloset al., 3D sensors for the HL-LHC JINST 12 (2017) C01026.

[9] N. Savic et al., Investigation of thin n-in-p planar pixel modules for the ATLAS upgrade JINST 12 (2016) C12008.

[10] A. Macchiolo et al., Optimization of thin n-in-p planar pixel modules for the ATLAS upgrade at HL-LHC JINST 12 (2017) C01024.

[11] N. Wermes, Depleted CMOS pixels for LHC proton?proton experiments Nucl. Instrum. Meth. A 824 (2016) 483. 